\title{
Модели оценки затрат на собственный капитал компаний на развивающихся рынках капитала
}

\author{
Дранев Ю.Я. ${ }^{21}$, Нурдинова Я.С. ${ }^{22}$, Редькин В.А. ${ }^{23}$, Фомкина С.А. $^{24}$
}

Одной из наиболее актуальных проблем корпоративных финансов является оценка затрат на собственный капитал компаний. Особенности развивающихся рынков капитала не всегда позволяют использовать традиционные методы оценки. Действительно, развивающимся рынкам капитала свойственны дополнительные риски, связанные с неэффективностью рынка, ограниченными возможностями диверсификаџии, а также институциональными особенностями развивающихся стран. Для учета этих рисков значительное количество исследователей разрабатывали новые модели оценки требуемой доходности на собственный капитал. Однако тестирование этих моделей часто приводило $\kappa$ противоречивым результатам.

Данная работа посвящена обзору статей, в которых проводятся эмпирические тесть и теоретический анализ наиболее известных моделей оценки затрат на собственный капитал.

\section{JEL: G12, G32}

Ключевые слова: САРМ, затраты на собственный капитал, развивающиеся рынки капитала, глобальная САРМ, локальная САРМ, гибридная САРМ

\section{Введение}

Ставка требуемой доходности находится в непосредственной зависимости от оценки рисков компании инвесторами и является одним из ключевых параметров при оценке ее стоимости. Несмотря на широкое применение показателя требуемой доходности, выбор наилучшего метода оценки затрат на собственный капитал на развивающихся рынках капитала до сих пор вызывает множество вопросов исследователей, Дело в том, что для развивающихся рынков капитала характерны дополнительные риски, оказывающие влияние на динамику требуемой доходности на собственный капитал.

Большинство исследователей пришли к выводу о том, что глобальная САРМ обладает рядом недостатков, особенно при использовании на развивающихся рынках капитала. Это обстоятельство привело к появлению различных модификаций модели, например, предложенных Шарпом (Sharpe,1964) и Линтнером (Lintner, 1965). Появились и способы определения затрат на собственный капитал, не основанные на САРМ. При этом исследователи получили противоречивые результаты тестирования моделей на развивающихся рынках.

В первой части работы рассмотрена классификация моделей затрат на собственный капитал. Вторая часть посвящена различным способам эмпирического анализа ожидаемой доходности на собственный капитал. В заключение будут проведены сопоставления результатов рассмотренных исследований и сделаны выводы относительно выбора модели оценки затрат на собственный капитал для развивающихся рынков капитала.

\footnotetext{
${ }^{21}$ Старший преподаватель кафедры экономики и финансов фирмы НИУ ВШЭ

${ }^{22}$ Студентка магистерской программы «Стратегическое управление финансами фирмы» НИУ ВШЭ

${ }^{23}$ Студент магистерской программы «Стратегическое управление финансами фирмы» НИУ ВШЭ

${ }^{24}$ Студентка магистерской программы «Стратегическое управление финансами фирмы» НИУ ВШЭ
} 


\section{Модели оценки затрат на собственный капитал}

В настоящее время существуют различные модели оценки затрат на собственный капитал, однако все они могут быть поделены на две большие группы (рис. 1): модели, основанный на САРМ, и иные.

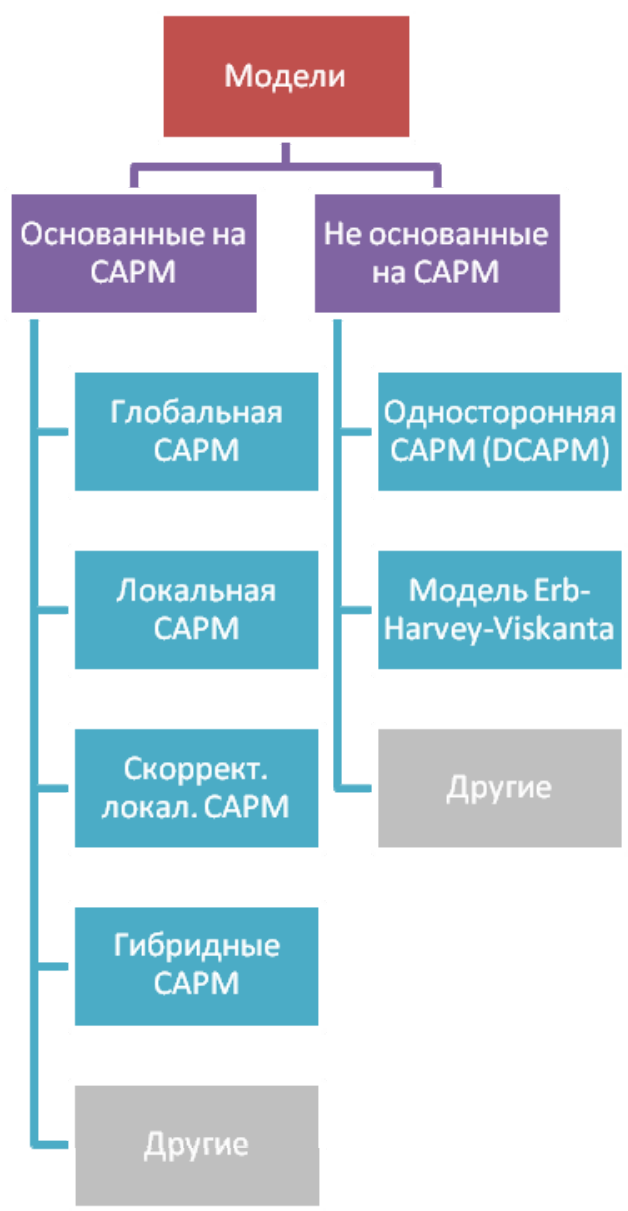

Рисунок 1. Классификация моделей оценки затрат на собственный капитал Источник: анализ авторов.

Вследствие резкого роста числа сделок, связанных со слияниями и поглощениями на развивающихся рынках капитала в конце 1990-х годов вопрос о верной оценке требуемой доходности и затрат на собственный капитал стал особенно актуальным. Реакцией на сложившуюся ситуацию стала статья Перейро (Pereiro, 2001), посвященная исследованию адекватности различных спецификаций модели САРМ. Аналогичной цели посвящены работы Сабал и фон Дженнера (Sabal, 2004; von Jenner,2008).

В своей работе Перейро делит модели на те, в основе которых лежит модель Шарпа и Линтнера, и те, что не основаны на САРМ. В таблице 1 представлены некоторые подходы к оценке требуемой доходности (Pereiro, 2001; Sabal, 2004; von Jenner, 2008).

Перейро отмечает, что довольно сложно защищать глобальную САРМ при условии несовершенства рынка. Кроме того, по его мнению, модель локальной САРМ переоценивает риск. Годфри и Эспиноза обратили внимание на то, что включение странового риска в модель САРМ ведет к двойному учету риска страны, так как его часть может быть представлена в рыночной премии за риск, а Эрб с соавторами (Erb et al., 1995) показали, что рыночный риск включает в себя компонент макроэкономического риска. 
Модели оценки затрат на собственный капитал

\section{Pereiro (2001)}

1. Глобальная САРМ.

2. Локальная САРМ.

3. Скорректированная локальная

CAPM (предложена Перейро в 2001 г.).

4. Скорректированная глобальная CAPM (предложена Перейро в 2001 г.).

5. Модель ГодфриЭспинозы ( 1996).

6. DCAPM

(Estrada, 2000).

7. Модель кредитного риска.

Источники: (Pereiro, 2001; Sabal, 2004; Von Jenner, 2008).

Чтобы исключить двойной учет риска, Перейро предложил следующую модель:

$r_{e}=r_{f G}+C R P+\beta_{L L} \cdot\left(r_{m L}-r_{f L}\right) \cdot\left(1-R^{2}\right)$

где $\mathrm{R}^{2}$ - коэффициент детерминации регрессионного уравнения между доходностью локального рынка акций и страновым риском, т.е. доля волатильности локального рынка, которая объясняется страновым риском.

Для решения проблемы рассчета долгосрочных рыночных премий и бет Перейро была введена скорректированная гибридная САРМ, которая модифицирует глобальную премию в локальную при помощи чувствительности доходностей акций локального рынка к доходностям глобального рынка:

$$
r_{e}=r_{f G}+C R P+B C_{L G} \beta_{G G} \cdot\left(r_{m G}-r_{f G}\right) \cdot\left(1-R^{2}\right),
$$

где:

$B C_{L G}$ - бета-коэффициент (относительная цена странового риска как угол наклона регрессии локального рынка акций к глобальному);

$\beta_{G G}$ - среднее значение безрычагового бета-коэффициента по сопоставимым компаниям на глобальном рынке (может использоваться и бета-коэффициент локального рынка);

$R^{2}$ - коэффициент детерминации регрессионного уравнения между локальным рынком акций и страновым риском.

Также одной из модификаций гибридных САРМ является модель Годфри-Эспинозы:

$$
r_{\varepsilon}=r_{f U S}+C R P+B A \cdot\left(r_{m U S}-r_{f U S}\right), B A=\frac{9}{v_{W S}} \cdot 0,6 \text {, }
$$

где коэффициент детерминации регрессионного уравнения равен 0,6 .

Международная САРМ, в дополнение к предпосылкам, лежащим в основе САРМ, предполагает наличие у инвесторов валютной потребительской корзины и выглядит следующим образом (Sercu and Uppal,1995):

$$
\begin{aligned}
& r_{e w x}=r_{f}+\beta_{i}\left(r_{m e}-r_{f}\right)+\gamma_{t x}\left(s_{x}+r_{x}-r_{f}\right), \\
& \text { где: } \\
& r_{w i x} \text { - доходность актива } i \text { в стране } x ;
\end{aligned}
$$


$r_{f}$ - безрисковая ставка в базовой валюте (например, в долларах США);

$\gamma_{i x}$ - бета-коэффициент безрисковой ставки;

$s_{x}-$ процент изменения обменного курса базовой валюты по отношению к национальной;

$T_{x}$ - безрисковая ставка в национальной валюте.

Однако бета-коэффициенты компаний на развивающихся рынках капитала весьма неустойчивы во времени (Harvey,1995; Estrada, 2000). Данный факт привел к созданию моделей оценки затрат на собственный капитал, не основанных на САРМ.

Эстрада предлагает по-новому взглянуть на проблему определения размера требуемой доходности, предложив учитывать реакцию инвесторов лишь при отрицательной динамике доходности активов. Автор разработал модель, где в качестве меры риска выступает:

$$
\beta_{i}=\frac{E\left[m \min \left(r_{i}-\mu_{i}, 0\right) \cdot \min \left(r_{\mathrm{m}}-\mu_{m}, 0\right)\right]}{E\left[\operatorname { m b n } \left(x_{m}\right.\right.}
$$

где $\mu_{2}$ и $\mu_{m}-$ средняя доходность рассматриваемой акции и рыночного портфеля, соответственно. Эта модель, получившая название «односторонняя CAPM (DCAPM)», более точно отражает восприятие риска инвесторами.

Pocc (Ross, 1976) предложил модель оценки затрат на собственный капитал (Arbitrage Pricing Theory - APT). В соответствии с моделью доходность актива линейно связана с набором факторов:

$$
r_{e n}=r_{t}+\beta_{i 1} f_{1}+\beta_{i 2} f_{2}+\cdots+\beta_{m} f_{n}
$$

где $f_{1}, f_{2}, \ldots, f_{n}$ - факторы, оказывающие влияние на доходность актива.

Для экономики без фондового рынка Эрб с соавторами (Erb et al., 1996) предложили иную модель:

$$
\gamma_{e t,+1}=\psi_{0}+\gamma_{1} \ln \left(C C D_{t, t}\right)+a_{t, t+1}
$$

где $C C R_{t, t}-$ кредитный рейтинг страны.

Сабал (Sabal,2004) большое внимание уделяет целесообразности включения премии за страновой риск в модели оценки затрат на собственный капитал. Автор отмечает, что страновой риск в основном ассоциируется с риском политическим. Вместе с тем премия за страновой риск определяется как спред доходности по государственным облигациям развитой страны и развивающейся. По мнению Сабал, включение такой премии за страновой риск теоретически необоснованно по нескольким причинам:

1. Страновой риск неодинаков для различных проектов.

2. CRP не полностью систематический: Сабал напоминает, что во многих случаях корреляция между доходностями активов на развитых рынках и доходностями на развивающихся рынках незначительна.

3. Кредитный риск - не эквивалент премии за страновой риск.

По мнению Сабал, в большинстве случаев этот показатель является лучшей грубой аппроксимацией, а в худшем - может полностью ввести в заблуждение. Мнение Сабал также поддерживает фон Дженнер (Von Jenner, 2008).

Перейро в уже упоминавшейся работе дает рекомендации относительно целесообразности применения тех или иных моделей при различной степени интеграции рынка, а также доступности и качества данных (таблица 2).

Перейро отмечает, что в случае, когда аналитик желает использовать модели, не основанный на САРМ, то ему, скорее всего, следует отдать предпочтение модели Эстрада. 
Степень интеграции рынка

\begin{tabular}{cccc}
\hline \multicolumn{1}{c}{ Надежность } \\
локальных данных \\
$\begin{array}{c}\text { для расчета премии } \\
\text { за риск и бета }\end{array}$ & Высокая & Высокая & Низкая \\
& Глобальная САРМ & $\begin{array}{c}\text { Локальная САРМ } \\
\text { Скорректированная } \\
\text { локальная САРМ }\end{array}$ \\
\hline
\end{tabular}

Источник: (Pereiro, 2001).

По мнению фон Дженнера, несмотря на то что самый распространенный способ оценивания требуемой доходности - локальная САРМ, есть ряд ограничений для применения данной модели:

- на развивающихся рынках не всегда существует безрисковая процентная ставка из-за низкой надежности государственных долговых бумаг;

- расчет бета может быть сопряжен с различными трудностями (поиск подходящей компании для расчета, низкая ликвидность, короткая история торгов).

С точки зрения Сабал, в дальнейшем локальная САРМ, вероятнее всего, исчезнет, а фон Дженнер заключает, что с точки зрения доступности данных наиболее применима модель home $\mathrm{CAPM}^{25}$.

Локальная САРМ и международная САРМ просты в применении, однако аналитик может столкнуться с трудностями в оценке некоторых параметров. Выбор между ними зависит от того, диверсифицирует ли инвестор свой портфель между странами. Если да, то международная модель САРМ подходит больше всего; если нет, то следует проводить расчеты при помощи локальной САРМ.

Фон Дженнер отмечает, что наиболее теоретически обоснованные модели - локальная САРМ, международная САРМ и мультифакторная модель, однако последняя из них сложна в применении.

Сабал рекомендует осуществлять оценку требуемой доходности, используя несколько наиболее подходящих по логике моделей для получения диапазона ставок, после чего можно принимать решение о ее размере.

Несмотря на теоретическое обоснование применения тех или иных моделей оценки затрат на собственный капитал на развивающихся рынках, обратим внимание на эмпирические тесты данных моделей.

\section{Эмпирический анализ зависимости ожидаемой доходности на собственный капитал от премии за рыночный риск}

Фама (Fama, 1976) предложил метод тестирования моделей, основанных на CAPM. Ключевым моментом в нем является оценка беты актива. Для этого на основе реализованных доходностей активов (ex-post) и рыночной доходности строится регрессия на временном ряде (time-series regression). Сама регрессия выглядит следующим образом:

\section{$\kappa_{t z}=a_{i}+s_{i} R_{m e}+s_{t r t}$}

Здесь бета определяется как оценка коэффициента при рыночной доходности. Часто оценивается модель, где переменными являются не доходность актива и рыночная доходность, а премии над безрисковой ставкой (Bruner et al., 2008), что является более корректным, поскольку предыдущая спецификация не учитывает безрисковой доходности, которая присутствует в САРМ.

Регрессии оцениваются для каждого актива и для каждой сравниваемой модели, после чего проводится анализ объясняющей силы каждой модели и делается вывод о том, какая из

\footnotetext{
${ }^{25}$ Схожа с локальной САРМ
} 
них больше подходит для данного рынка.

В случае, когда модель оценивается в отклонениях от безрисковой ставки, отдельное внимание нужно уделить значимости константы в регрессии (см. регрессию, представленную в начале этого раздела). Данная константа интерпретируется как некая избыточная доходность, которую не объясняет модель САРМ. Бартольди и Пир (Bartholdy and Peare, 2003) утверждают, что, во-первых, САРМ может быть неверно специфицированной моделью - так, другими исследователями уже были предложены альтернативные модели - АРТ и трехфакторная модель Фамы и Френча (Fama, French, 1992). Во-вторых, даже если САРМ корректна, ${ }^{a_{i}}$ может заключать в себе эффекты смещения беты, поскольку в разные периоды бета может меняться. Еще одна причина, по которой может быть отвергнута гипотеза о равенстве константы нулю в CAPM (Diwani, 2010) - техническая.

Таким образом, проверка гипотезы о равенстве константы нулю по сути является проверкой, объясняющей силы модели САРМ. Единое мнение о равенстве константы нулю не достигнуто: например, Йошино с соавторами, а также Хасан с соавторами (Yoshino et al., $2009 ;^{26}$ Hasan et al., 2011 ${ }^{27}$ ) получили результаты, отвергающие нулевую гипотезу, и приходят к выводу о несостоятельности локальной САРМ, в то время как Дивани и Михайлидис с соавторами (Diwani,2010, ${ }^{28}$ Michailidis et al., 2006 ${ }^{29}$ ) подтверждают незначимость константы и ее соответствие нулевому значению.

Помимо этого, некоторые исследователи фокусируются на тестировании линейности CAPM (Ocampo, 2004; Michailidis et al., 2006; Iqbal, Brooks, 2007; Olakojo, Ajide, 2010; Choudhary, Choudhary, 2010; Reddy, Thomson, 2011). При этом часть исследователей отвергают гипотезу о линейной зависимости (Iqbal, Brooks, 2007; Olakojo, Ajide, 2010).

Для компаний с развивающихся рынков, которые, между тем, ведут свою деятельность и на других рынках Брюнер с соавторами (Bruner et al., 2008) предложили включать в регрессию дополнительный фактор: премию за риск на других рынках. В своей работе они доказали, что оба фактора являются значимыми, то есть можно говорить о наличии интеграциями между рынками, а величина коэффициентов при этом показывает степень влияния каждого из них на затраты на капитал для конкретной компании.

Тем не менее даже лучшая из моделей САРМ обладает достаточно низкой объясняющей силой, поэтому часто авторы вводят в модель дополнительные факторы (Banz, 1981; Fama, French, 1992). Сравнение арбитражной модели Росcа (Ross, 1976) и различных модификаций CAPM невозможно в рамках описанного выше подхода. Поэтому строится сквозная регрессия (cross-sectional regression), где в качестве зависимой переменной выступают также доходности компаний (либо премии над безрисковой доходностью), а в качестве объясняющих переменных берутся те факторы, которые могут оказывать влияние на величину затрат на капитал (в САРМ одним из таких факторов является бета актива) (Febrian, Herwany, 2010).

Следует отметить, что существуют различные подходы к определению рыночной доходности, используемой в САРМ. Часто авторы исследовательских работ используют в этой роли доходность какого-либо индекса. В тоже время, Брюнер с соавторами определили рыночную доходность как взвешенную по объему размещенных акций доходность этих компаний. Используя такой метод, они получили, что на развивающихся рынках капитала использование локальной модели является более предпочтительным по сравнению с глобальной, тогда как на развитых рынках обе модели дают идентичный результат. Введение отраслевого фактора улучшало модель лишь в $20 \%$ случаев.

\footnotetext{
${ }^{26}$ Выборка: 24 компании, Бразилия, 1998-2006 годы

${ }^{27}$ Выборка: 80 компаний, Бангладеш, 2005-2009 годы

${ }^{28}$ Выборка: 28 компаний, Индия, 2004-2009 годы

${ }^{29}$ Выборка: 100 компаний, Греция, 1998-2002 годы
} 


\section{Эмпирический анализ зависимости ожидаемой доходности на собственный капитал от показателей риска}

Ряд авторов предлагают иной способ регрессионного анализа - построение парных и множественных регрессий, в которых в качестве зависимой переменной выступает средняя доходность, а в качестве объясняющих - показатели риска (Бухвалов, Окулов, 2006; Estrada, 2007; Foong, Goh, 2010; Teplova, Shutova, 2011). Кроме того, в качестве регрессора может использоваться показатель смещения.

При тестировании модели DCAPM Эстрада (Estrada, 2007) рассмотрел выборку из 23 развитых и 27 развивающихся стран (в их число вошла и Россия) в период с 1988 по 2001 год и пришел к выводу, что для развивающихся рынков модель DCAPM имеет большую статистическую и экономическую значимость, чем локальная САРМ. Аналогичный результат получили Бухвалов и Окулов на российской выборке (период 1996-2002 годы). Однако авторы отмечают, что DCAPM все же обладает небольшой объясняющей силой.

Средняя доходность российских компаний в период 2004-2010 годов рассматривались также в работе Тепловой и Шутовой . Результатом работы стал вывод о том, что модель DCAPМ имеет низкую объясняющую способность.

Фунг и Гох на основе данных рынка капитала Малайзии за 2000-2007 годы провели оценку нескольких показателей риска доходности акций, а также предложили еще один вариант двухфакторной модели:

$$
r_{e i}=r_{f}+\beta_{L i} \cdot\left(r_{m}-r_{f}\right)+\beta_{G i} \cdot\left(r_{M}-r_{F}\right),
$$

где:

$$
\begin{aligned}
& r_{f} \text { - локальная безрисковая ставка; } \\
& r_{F} \text { - глобальная безрисковая ставка; } \\
& r_{m} \text { и } r_{M}-\text { доходности локального и глобального рыночных портфелей }
\end{aligned}
$$
соотвественно; $\quad \beta_{L t} \beta_{Q}-$ чувствительность компаний к локальным и глобальным риск-факторам.

В качестве мер риска авторами были рассмотрены: среднеквадратическое отклонение доходности компании, локальная бета, глобальная бета, беты двухфакторной модели, а также односторонняя модификация всех указанных показателей. Фунг и Гох проранжировали их по степени их объясняющей силы (таблица 3).

Таблица 3

Объясняющая сила мер риска

\begin{tabular}{cccccccc}
\hline Модель & $\mathrm{R} 2$ & $\mathrm{AdjR} 2$ & $\begin{array}{c}\text { Log } \\
\text { Likelihood }\end{array}$ & AIC & SC & Ранг \\
Полуотклонение & 0,4363 & 0,3546 & $-22203,33$ & 10,2192 & 11,0309 & 1 \\
Односторонние беты & 0,3680 & 0,2763 & $-22458,09$ & 10,3340 & 11,1472 & 2 \\
двухфакторной модели & & & & & & \\
Бета & 0,3630 & 0,2706 & $-22475,97$ & 10,3416 & 11,1533 & 3 \\
Беты двухфакторной модели & 0,3627 & 0,2701 & $-22476,91$ & 10,3424 & 11,1556 & 4 \\
Глобальная бета & 0,3599 & 0,2672 & $-22486,47$ & 10,3463 & 11,1580 & 5 \\
Стандартное отклонение & 0,3592 & 0,2664 & $-22488,94$ & 10,3474 & 11,1591 & 6 \\
Односторонняя бета & 0,3583 & 0,2653 & $-22492,14$ & 10,3488 & 11,1606 & 7 \\
Глобальная односторонняя бета & 0,3582 & 0,2652 & $-22492,47$ & 10,3490 & 11,1607 & 8 \\
\hline
\end{tabular}

Источник: (Foong, Goh, 2010).

Фунг и Гох заключили, что полученные результаты подтверждают мнение Эстрада (Estrada, 2007) относительно преимущества односторонних мер риска над стандартными показателями. 
Другие способом тестирования является деление выборки на портфели (Estrada, 2007; Galagedera, 2009). В рамках данного метода выборка ранжируется по величине коэффициента бета и разбивается на основе этого на три портфеля: с высоким риском, со средним риском и с низким риском. После этого находится разность между бетой портфеля с высоким риском и бетой портфеля с низким риском. Полученный спред сравнивается со спредом доходности по этим портфелям.

\section{Тестирование моделей оценки затрат на собственный капитал на различных временных отрезках}

Модель САРМ, обладает одним очень существенным недостатком, а именно - она статична, и результаты, полученные с помощью САРМ, вполне могут оказаться неустойчивыми.

Поэтому актуальным становится иное направление регрессионного анализа. Оно заключается в том, что выборка разбивается на два периода: базовый и контрольный (Mongrut et al., 2010). По базовому периоду с помощью регрессионного анализа оценивается коэффициент бета, после чего предсказанные значения доходности сравниваются с наблюдаемыми значениями доходности.

В работе Монграта с соавторами (Mongrut et al., 2010) рассматривалась выборка из 32 компаний Балтики в период 2000--2008 годов, и полученные по DCAPM оценки оказались близки к наблюдаемым.

Выборка может делиться на два и более периода, на каждом из которых модель оценивается отдельно.

Довольно часто авторы выделяют докризисный, кризисный и посткризисный периоды (Barclay et al., 2010; Febrian, Herwany, 2010), другие же авторы просто делят выборку примерно на равные части (Bruner, 2008).

Однако и здесь единство результатов не было достигнуто: в частности, Bruner et al., 2008) пришли к выводу, что коэффициент бета является достаточно стабильным во времени, a результаты сравнения различных моделей являются постоянными и не зависят от рассматриваемого периода ${ }^{30}$. С другой стороны, Фебриан и Хэвани, сравнивающие локальную САРМ и АРТ на рынке капитала Индонезии для трех различных периодов: перед финансовым кризисом, в течение кризиса и после него ${ }^{31}$, приходят к выводу, что АРТ более эффективна для индонезийского рынка по сравнению с САРМ. С этой точки зрения многофакторная модель является более подходящей в период кризиса, когда в экономике высокая инфляция и сильная волатильность обменного курса. Однако Фебриан и Хэвани отмечают, что адекватность модели АРТ зависит от точности выбора макроэкономических факторов.

Вместе с тем Барклай с соавторами сделали принципиально иные выводы. Авторы протестировали глобальную и локальную САРМ, а также их модификации с включением дополнительных факторов (в том числе валютных факторов). Выборка включила данные по 20 развивающимся странам, разделенные на два субпериода: 1995-2001 годы и 20022008 годы. Оказалось, что на разных субпериодах следует использовать разные модели оценки затрат на собственный капитал. В течение первого (докризисного и кризисного) периода при анализе развивающихся рынков локальная САРМ превосходит глобальную модель, тогда как в посткризисный период глобальная САРМ показывает лучшие результаты.

В отличие от рассмотренных выше работ Коркмаз с соавторами (Korkmaz et al., 2010) используют совсем другой подход: он строит модель со встроенным Марковским процессом, который обеспечивает переключение модели САРМ в два режима в зависимости от волатильности остаточного члена регрессии. Модель с Марковским процессом учитывает

\footnotetext{
${ }^{30}$ Напомним, что в работе сравнивались глобальная и локальная САРМ

${ }^{31}$ Общий период: июль 1992 - июнь 2007 года
} 
реакцию рынка на различные шоки в экономике. В ходе оценки было получено, что такая модель обладает большей объясняющей силой по сравнению со стандартной САРМ. Коркмаз с соавторами приходят к выводу, что САРМ занижает систематический риск в периоды с высокой волатильностью и завышает в периоды с низкой.

Другой способ учета влияния времени на изменение доходности - включение в модель дополнительного фактора, определяемого Кархарт (Carhart, 1997) как средняя доходность двух портфелей, показавших высокую доходность в предыдущем периоде, минус средняя доходность двух портфелей, показавших низкую доходность. Купер с соавторами (Cooper et al., 2004) показывает, что если этот фактор оказывается значимым, то фактически это свидетельствует о влиянии деловых циклов на доходность акций.

\section{Заключение}

Большинство авторов приходят к выводу о том, что на развивающихся рынках капитала локальная САРМ более эффективна, чем глобальная модель, в то время как на развитых рынках между ними практически нет разницы. Однако применение локальной САРМ может привести к двойному учету странового риска при расчете исследуемого показателя, в связи с чем предлагаются корректировки расчетного показателя. Развивающие рынки капитала часто характеризуются ненадежностью необходимых данных, что привело к появлению гибридных модификаций САРМ, использование которых осложняется применением в качестве прокси для страновой премии спреда доходности по гособлигациям. Эмпирические тесты, направленные на проверку линейной зависимости между компонентами САРМ и равенства константы в регрессии нулю, не дают однозначного ответа.

Многофакторные САРМ или модель АРТ показывают в целом более высокую объясняющую силу, чем любая однофакторная модель САРМ. Однако многофакторные модели также обладают рядом недостатков.

Таким образом, выбор той или иной модели оценки затрат на капитал в эмпирическом исследовании должен зависеть от требуемого уровня ее объясняющей силы. Так, САРМ в своей классической форме дает прогноз, опираясь лишь на рыночную премию за риск. Если же предполагается, что данные о рыночной доходности не отражают в себе все факторы риска, влияющие на деятельность компании, то наиболее приемлемым будет использование многофакторных моделей.

Необходимо отметить, что в последние годы внимание исследователей в значительной степени обращено к проверке эффективности моделей, учитывающих реакцию инвесторов лишь при снижении доходности активов (в частности, DCAPM). Однако тесты подобных модификаций на выборках развивающихся стран также дают противоречивые результаты.

Учитывая вышесказанное, можно сделать вывод о том, что не следует ограничивать выбор на одном из способов определения величины требуемой доходности актива, а стоит применить несколько методов и провести сравнение полученных результатов.

\section{Список литературы}

1. Бухвалов, А.В., Окулов, В.Л. Классические модели ценообразования на капитальные активы и российский финансовый рынок. Часть 2. Возможность применения вариантов модели САРМ // Научные доклады. 2006 № 36(R). СПб.: НИИ менеджмента СПбГУ.

2. Banz, R.W. (1981), The Relationship between Return and Market Value of Common Stocks, Journal of Financial Economics, 1(9) (1981) 3-18.

3. Barclay, R., Fletcher, J., Marshall, A. (2010), Pricing Emerging Market Stock Returns: An Update, Emerging Markets Review, 11 (2010) 49-61.

4. Bartholdy, J., Peare, P. (2003), Unbiased Estimation of Expected Return Using CAPM , 
International Review of Financial Analysis, 12 (2003) 69-81.

5. Bruner, R., Li, W., Kritzman, M. (2008), Market Integration in Developed and Emerging Markets: Evidence from the CAPM, Emerging Markets Review, 9 (2008) 89-103.

6. Carhart, M.M. (1997), On Persistence in Mutual Fund Performance, Journal of Finance, 52 (1997), 57-82.

7. Choudhary, K., Choudhary, S. (2010), Testing Capital Asset Pricing Model: Empirical Evidences from Indian Equity Market. Eurasian Journal of Business and Economics, 3(6) (2010) 127-138.

8. Cooper, M.J., Gutierrez JR., R.C., Hameed, A. (2004), Market States and Momentum, The Journal of Finance, 59(3) (2004), 1345-1365.

9. Diwani, M. (2010), Testing the CAPM in the Indian Market, Lund University, February: http://www.lunduniversity.lu.se/o.o.i.s?id=24965\&postid $=1583204$

10. Erb, C.B., Harvey, C.R., Viskanta, T.E. (1995), Country Risk and Global Equity Selection, The Journal of Portfolio Management, 22(3) (1996) 46-58.

11. Estrada, J. (2000), The Cost of Equity in Emerging Markets: a Downside Risk Approach, Emerging Markets Quarterly, 4 (2000) 19-30.

12. Estrada, J. (2007), Mean-semivariance Behavior: Downside Risk and Capital Asset Pricing. International Review of Economics and Finance, 16 (2007), 169-185.

13. Fama, E.F. (1976), Foundations of Finance. New York, Basic Books.

14. Fama, E.F., French, K.R. (1992), The Cross-Section of Expected Stock Returns.Journal of Finance, 47(2) (1992) 427-465.

15. Febrian, E., Herwany, A. (2010), The Performance of Asset Pricing Models Before, During, and After an Emerging Market Financial Crisis: Evidence from Indonesia, The International Journal of Business and Finance Research, 4(1) (2010), 85-97.

16. Foong, S.-S., Goh, K.-L.(2010), Measuring the Cost of Equity of Emerging Market Firms: The Case of Malaysia, Asian Academy of Management Journal of Accounting and Finance, 6(1) (2010) 25-46.

17. Galagedera, D.U.A. (2009), Economic Significance of Downside Risk in Developed and Emerging Markets, Applied Economics Letters, 16(16) (2009) 1627-1632.

18. Godfrey, S., Espinosa, R. (1996), A Practical Approach to Calculating Costs of Equity for Investments in Emerging Markets, Journal of Applied Corporate Finance, 6 (3), 80-90.

19. Harvey, C.R. (1995), Predictable Risk and Returns in Emerging Markets, Review of Financial Studies, 8 (1995) 773-816.

20. Hasan, M.Z., Kamil, A.A., Mustafa, A., Baten, M.A. (2011), A Validity Test of Capital Asset Pricing Model for Dhaka Stock Exchange, Journal of Applied Sciences, 11(20) (2011) 3490-3496.

21. Von Jenner, M.H. (2008), Calculating the Cost of Equity in Emerging Markets, JASSA, The Finsia Journal of Applied Finance, 4 (2008) 21-25.

22. Iqbal, J., Brooks, R. (2007), A Test of CAPM on the Karachi Stock Exchange, International Journal of Business, 12(4) (2007) 429-444.

23. Korkmaz, T., Ismail, E., Gu:rkan, S. (2010), Testing of the International Capital Asset Pricing Model with Markov Switching Model in Emerging Markets, Investment Management and Financial Innovations, 7(1) (2010) 37-49.

24. Lintner, J. (1965), The Valuation of Risk Assets and the Selection of Risky Investments in Stock Portfolios and Capital Budgets, The Review of Economics and Statistics, 47(1) (1965) 13-37.

25. Michailidis, G., Tsopoglou, S., Papanastasion, D., Mariola, E. (2006), Testing the Capital Asset Pricing Model (CAPM): The Case of the Emerging Greek Securities Market, International Research Journal of Finance and Economics, 4 (2006) 78-91.

26. Mongrut, S., Paškevičius, A., Dubinskas, P., Kovalevskaja, R., Fuenzalida, D. (2010), Estimating the Cost of Equity in the Baltic Region, Ekonomika, 89(3) (2010) 40-54.

27. Ocampo, P.B.D. (2004), Alternative Methodologies for Testing CAPM in the Philippines 
Equities Market, Philippine Management Review, 11 (2004) 46-54.

28. Olakojo, S.A., Ajide, K.B. (2010), Testing the Capital Asset Pricing Model (CAPM): The Case of the Nigerian Securities Market, International Business Management, 4(4) (2010) 239-242.

29. Pereiro, L.E. (2001), The Valuation of Closely-Held Countries in Latin America, Emerging Markets Review, 2 (2001) 330-370.

30. Ross, S.A. (1976), The Arbitrage Theory of Capital Asset Pricing, Journal of Economic Theory, 13 (1976) 341-360.

31. Reddy, T.L., Thomson, R.J. (2011), The Capital Asset Pricing Model: the Case of South Africa, South African Actuarial Journal, 11 (2011) 43-84.

32. Sabal, J. (2004), The Discount Rate in Emerging Markets: A Guide, Journal of Applied Corporate Finance, , 16(2-3) (2004) 155-166.

33. Sercu, P., Uppal, R., (1995), International Financial Markets and The Firm. Cincinnati, Ohio: College Division, South-Western Pub.

34. Teplova, T., Shutova, E. (2011), A Higher Moment Downside Framework For Conditional And Unconditional CAPM In The Russian Stock Market, Eurasian Economic Review, 1(2) (2011) 157-178.

35. Yoshino, J.A., Santos, E.B. (2009), Is the CAPM Dead or Alive in the Brazilian Market?, Review of Applied Economics, 5(1-2) (2009), 127-142. 